\title{
Erratum: Reconciling Coulomb breakup and neutron radiative capture [Phys. Rev. C 96, 015801 (2017)]
}

\author{
P. Capel and Y. Nollet
}

(Received 31 May 2018; published 16 July 2018)

\section{DOI: 10.1103/PhysRevC.98.019906}

The idea to infer radiative-capture cross sections of astrophysical interest, viz. at low energy, from Coulomb-breakup measurements was first suggested by Baur et al. [1]. The latter reaction can be seen as an exchange of virtual photons between the initially bound projectile and the target and hence as the time-reversed reaction of the former in which the nucleus is synthesized by the fusion of two clusters and the emission of a photon. At the first order of the perturbation theory, it is possible to directly relate the Coulomb-breakup cross section to the radiative-capture one by a detailed balance [1-3]. Because breakup measurements can be performed at higher energies where the cross sections are higher and therefore easier to measure, many breakup experiments have been performed following this idea. Unfortunately, it has been shown later that significant higher-order effects in the breakup process spoil this nice picture [4,5].

In Refs. [6,7], Summers and Nunes have suggested comparing accurate breakup calculations, i.e., including both the nuclear and the Coulomb interactions between the projectile and the target at all orders, to experimental data and to extract from this comparison the asymptotic normalization constant (ANC) for the bound state of the nucleus under investigation. This ANC can then be used to constrain radiative-capture calculations. They have tested their idea on the particular case of ${ }^{15} \mathrm{C}$ for which accurate measurements have been performed for both its Coulomb breakup [8] and its synthesis by radiative capture [9]. Their radiative-capture calculations, constrained by the Coulomb-breakup data, are in seemingly good agreement with the direct measurement [6,7]. A similar agreement has been obtained by Esbensen using a different model of the breakup reaction [10].

In the original paper, we have pushed this idea further and studied its sensitivity to the description of the ${ }^{14} \mathrm{C}-n$ continuum. Albeit smaller than the role of the ANC of the ground state, this part of the description of the nucleus is not negligible as shown earlier in Ref. [11]. However, following Refs. [12,13], it is possible to account for this effect by selecting the breakup data at forward angle and low energy in the projectile continuum (see the original paper). In this way, it seemed that even better agreement could be obtained between direct and Coulomb-breakup estimates.

This excellent agreement was obtained following the prescription of Summers and Nunes to rescale the low-energy data point, assuming it corresponded to a Maxwellian average on the neutron energy. Unfortunately, as pointed out by Esbensen and Reifarth [14], this is not the case. To be properly compared to the capture measurements, calculations should be averaged over the energy distribution of the neutron beams used in the experiment [15] (see Fig. 3 of Ref. [9]).

In this Erratum, we perform this averaging in a systematic way for the $12{ }^{14} \mathrm{C}-n$ potentials developed for our analysis in the original paper. The results are summarized in Table I. Each line of that table corresponds to one potential identified by its geometry in the $s$ partial wave of the ${ }^{15} \mathrm{C}$ ground state and in the $p$ wave of the ${ }^{14} \mathrm{C}-n$ continuum (see Sec. III A of the original paper for more details). The numbers in the various columns provide the radiative-capture cross-section $\sigma_{n, \gamma}$ obtained from averaging the theoretical prediction over the energy distribution of the neutron beams used in the experiment of Reifarth et al. [9]. For each of the beam energies considered $(E=23.3,150,500$, and $750 \mathrm{keV})$ we provide the value obtained with the bare potentials of our original paper (denoted "unscaled") and the "scaled" cross sections resulting from the multiplication of the unscaled result by the factor obtained from the fit of our breakup calculation with the data of Nakamura et al. [8] (see our original paper for details). That scaling is performed at low energy $(E<0.5 \mathrm{MeV})$ and forward scattering angles $\left(\Theta<2.1^{\circ}\right)$, which was found to be the most accurate one, as it focuses on the energy range of astrophysical interest and the angles at which the nuclear interaction between the projectile and the target is less significant, i.e., where the process is fully dominated by the Coulomb interaction (see our original paper). The fourth line before the last one provides the average and standard deviation over the 12 potentials. As already mentioned in the original paper, we see that once scaled all potentials provide nearly identical radiative-capture cross sections despite displaying very different unscaled cross sections. Accordingly, the standard deviation of the scaled results is tremendously reduced compared to that obtained for the unscaled results.

To properly confront these estimates to the experimental data (last line of Table I), we add to that main contribution the cross section for the capture to the $5 / 2^{+}$bound excited state of ${ }^{15} \mathrm{C}$, which we had neglected in our original paper. We describe this state as a $0 d 5 / 2$ neutron bound to the ${ }^{14} \mathrm{C}$ ground state and consider the same $12{ }^{14} \mathrm{C}-n$ potentials as in the original paper. Similar to the capture to the ground state, the dominant sensitivity comes from this excited-state ANC, the description of the $p$ continuum leads to only 5\% uncertainty. The estimate provided in the third to last line of Table I corresponds to the most usual geometry of the potential $\left(a_{p}=a_{s}=0.6 \mathrm{fm}\right)$. As already seen in Refs. [9,10], that contribution amounts to a mere 5\% of the total cross section; a rough estimate is therefore sufficient here. 
TABLE I. Theoretical radiative-capture cross sections obtained with the $12{ }^{14} \mathrm{C}-n$ potentials of the original paper averaged over the energy distribution of the neutron beam used in the experiment of Ref. [9]. The measured values are listed in the last row.

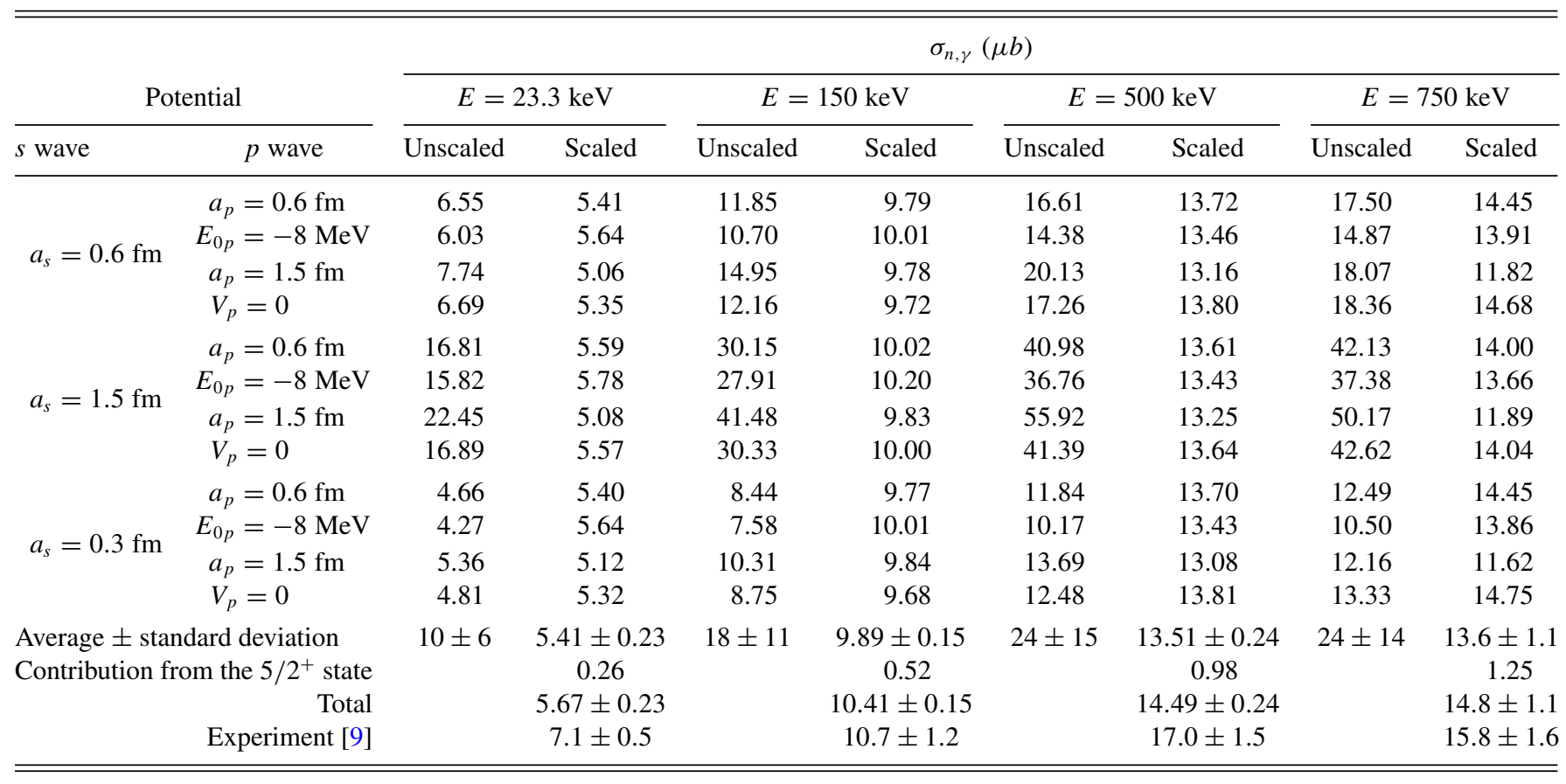

The total of these two contributions is shown in the penultimate line of Table I. Thanks to the scaling suggested in our original paper, this total is close to the experimental value. However, contrary to the results discussed in our original paper, we observe a systematic underestimation of the experiment by the theory prediction, similar to the one obtained by Esbensen [10]. Although our scaling method enables us to account for the influence of both the ANC and the continuum description and hence reduce the uncertainty in extracting the radiative-capture cross section from Coulomb breakup measurements, it does not fully reconcile both methods.

We thank C. Forssèn and R. Reifarth for interesting discussions on this averaging problem and for providing us with the numerical estimate of the neutron energy distributions. This project has received funding from the European Union's Horizon 2020 research and innovation programme under Grant Agreement No. 654002, the PRISMA Cluster of Excellence, and the Deutsche Forschungsgemeinschaft through the Collaborative Research Center 1044. P.C. is supported by the State of Rhineland-Palatinate.

[1] G. Baur, C. A. Bertulani, and H. Rebel, Nucl. Phys. A 458, 188 (1986).

[2] G. Baur and H. Rebel, Annu. Rev. Nucl. Part. Sci. 46, 321 (1996).

[3] G. Baur, K. Hencken, and D. Trautmann, Prog. Part. Nucl. Phys. 51, 487 (2003).

[4] H. Esbensen, G. F. Bertsch, and K. A. Snover, Phys. Rev. Lett. 94, 042502 (2005).

[5] P. Capel and D. Baye, Phys. Rev. C 71, 044609 (2005).

[6] N. C. Summers and F. M. Nunes, Phys. Rev. C 78, 011601 (2008).

[7] N. C. Summers and F. M. Nunes, Phys. Rev. C 78, 069908(E) (2008).

[8] T. Nakamura, N. Fukuda, N. Aoi, N. Imai, M. Ishihara, H. Iwasaki, T. Kobayashi, T. Kubo, A. Mengoni, T. Motobayashi,
M. Notani, H. Otsu, H. Sakurai, S. Shimoura, T. Teranishi, Y. X. Watanabe, and K. Yoneda, Phys. Rev. C 79, 035805 (2009).

[9] R. Reifarth, M. Heil, C. Forssén, U. Besserer, A. Couture, S. Dababneh, L. Dörr, J. Görres, R. C. Haight, F. Käppeler, A. Mengoni, S. O’Brien, N. Patronis, R. Plag, R. S. Rundberg, M. Wiescher, and J. B. Wilhelmy, Phys. Rev. C 77, 015804 (2008).

[10] H. Esbensen, Phys. Rev. C 80, 024608 (2009).

[11] P. Capel and F. M. Nunes, Phys. Rev. C 73, 014615 (2006).

[12] S. Typel and G. Baur, Phys. Rev. Lett. 93, 142502 (2004).

[13] S. Typel and G. Baur, Nucl. Phys. A 759, 247 (2005).

[14] H. Esbensen and R. Reifarth, Phys. Rev. C 80, 059904(E) (2009).

[15] C. Forssén and R. Reifarth (private communications). 